\title{
Haptic search with finger movements: using more fingers does not necessarily reduce search times
}

\author{
K. E. Overvliet · J. B. J. Smeets $\cdot$ E. Brenner
}

Received: 28 August 2006 / Accepted: 21 May 2007 / Published online: 12 June 2007

(C) Springer-Verlag 2007

\begin{abstract}
Two haptic serial search tasks were used to investigate how the separations between items, and the number of fingers used to scan them, influence the search time and search strategy. In both tasks participants had to search for a target (cross) between a fixed number of nontargets (circles). The items were placed in a straight line. The target's position was varied within blocks, and interitem separation was varied between blocks. In the first experiment participants used their index finger to scan the display. As expected, search time depended on target position as well as on item separation. For larger separations participants' movements were jerky, resembling 'saccades' and 'fixations', while for the shortest separation the movements were smooth. When only considering time in contact with an item, search times were the same for all separation conditions. Furthermore, participants never continued their movement after they encountered the target. These results suggest that participants did not use the time during which they were moving between the items to process information about the items. The search times were a little shorter than those in a static search experiment (Overvliet et al. in Percept Psychophys, 2007a), where multiple items were presented to the fingertips simultaneously. To investigate whether this is because the finger was moving or because only one finger was stimulated, we conducted a second experiment in which we asked participants to put three fingers in line and use them together to scan the items. Doing so increased the time in contact with the items for all separations, so search times were presumably longer in the
\end{abstract}

K. E. Overvliet ( $\square)$ · J. B. J. Smeets · E. Brenner

Faculty of Human Movement Sciences,

Vrije Universiteit, Van der Boechorststraat 9,

1081 BT Amsterdam, The Netherlands

e-mail: krista.overvliet@gmail.com static search experiment because multiple fingers were involved. This may be caused by the time that it takes to switch from one finger to the other.

Keywords Touch · Fingers - Movement - Orientation · Psychophysics $\cdot$ Human

\section{Introduction}

In most experiments on haptic perception in which a search paradigm was used, participants were asked to find a target amongst items presented to several fingertips simultaneously. Participants had to lower the index, middle and ring fingers of both hands onto the stimuli. They then had to keep their fingers in contact with all the stimuli until they found the target (Klatzky and Lederman 1995; Lederman and Klatzky 1997; Overvliet et al. 2007a, b; Purdy et al. 2004). This proved to be a difficult task, in which the target did not "pop out": in most experiments the search times were much longer when more fingers were used. Perhaps it is difficult because in daily life you usually touch objects sequentially while moving your finger(s) over their surfaces, instead of touching multiple objects with the fingers of a static hand. If so, the efficiency of tactile processing may be underestimated by such experiments.

There are two reasons why efficiency may be higher for a moving hand than for a passive hand. In the first place, when participants move their fingers over the stimulus, perception itself might get faster. A study by Philips, Johnson and Browne (1983) revealed that tactile letter recognition was consistently better when the letters were scanned than for stationary touch. Heller (1984) also found that active touch resulted in better recognition rates than either passive 
sequential or passive static touch. In the static haptic search tasks mentioned in the previous paragraph participants were very constrained in their movements. Giving them more freedom to explore the items could lead to shorter search times per item.

In the second place, having space between the items might increase the efficiency of the search in terms of the time spent on an item. You may use the time in which the finger is moving to a next item to process the previous item and decide whether this was the item you were looking for. There are two possible ways in which one could process tactile information when scanning a display. If information has to be fully processed before a movement is initiated to gather new information, the time spent moving between the items will influence the resulting search times: the larger the separation between the items, the longer the total search time. The time in contact with each item will be the same for all separations. However, if the information that has been gathered at the fingertips can be processed while moving on to the next item, and a new movement can be planned and initiated while the brain is still processing the gathered information, the time that it takes to move between the items can be used to process information. Longer separations between items could then result in shorter contact times with the items, leaving the total search time constant. There is some evidence that this happens in visual search tasks. In a study by Hooge and Erkelens (1996) participants had to search for a target by scanning a display with their eyes, and had to indicate the target by fixating it. In their study there were trials in which the target was only recognized after an eye movement away from the target had been made. The eyes then returned to the target to indicate that the target had been found. Liesker et al. (2007) found similar results in a task in which participants moved a small viewing window manually across the stimulus. On many trials participants passed the target and then had to return. The participants in both experiments must still have been processing information about the item while planning or executing the movement to the next item.

\section{Experiment 1}

To examine whether dynamic search is more efficient than static search, we designed a search task in which the participants had to scan a line of raised items with their fingertips. We varied the location of the target within blocks and the separation between the items between blocks. If moving the finger across the items speeds up the recognition of the items we will find shorter search times and less increase with the number of scanned items in this experiment, than in a previous static haptic search experiment using the same items (Overvliet et al. 2007a). If participants can use the time between items to process information about the item that they just passed, search times for the different separation conditions will be similar.

\section{Method}

\section{Participants}

Eight participants, four male and four female, with a mean age of 28.6 (range 25-41) participated in this experiment. Two of them stated that they were left-handed. Participants used their preferred hand to perform the task.

\section{Stimuli and apparatus}

The stimuli were made of $Z Y^{\circledR}$-TEX2 Swell paper by using the ZY ${ }^{\circledR}$-FUSE heater (Zychem Ltd., Cheshire, England). They consisted of circles and crosses with a line width of $1.4 \mathrm{~mm}$, which protruded about $1 \mathrm{~mm}$ from the surface of the paper. The size of the items was about $60 \%$ of the width of the individual participant's fingertip size; giving item widths of $8.5,9.6$ or $10.8 \mathrm{~mm}$. An earlier study (Overvliet et al. 2007a) has shown that it is quite difficult to distinguish between these items, with search times of $580 \pm 54 \mathrm{~ms}$ per item. The separation between the centres of the items was also scaled to fingertip size: it could be two, four or six times the item width. The different separations were measured in three separate blocks of trials. The items were positioned on a straight line, so that the participant could easily find them. Each sheet of swell paper contained five rows of items with identical spacing (for five trials). Ten sheets were presented, in random order, so that there were fifty trials for each separation condition. The target could appear at any position except the first one. In $25 \%$ of the trials there was no target. The movement of the participant's fingertip was measured with an OPTOTRAK ${ }^{\circledR}$ tracking system. This system measured the three-dimensional coordinates of an infrared emitting diode (IRED). An IRED was attached to the centre of the nail of the index finger of the participants' dominant arm. A curtain was placed between the participant and the stimuli to prevent the participant from seeing the items. The setup is shown in Fig. 1.

\section{Procedure}

We started by measuring the width of each participant's fingertip and preparing stimuli with appropriate dimensions. Participants were then asked to place the index finger of their dominant arm at the starting position (a dot) to the left of a row of items. Their task was to search for the target (a cross) amongst the distractors (circles) by scanning the row of items. A starting signal indicated that they could 


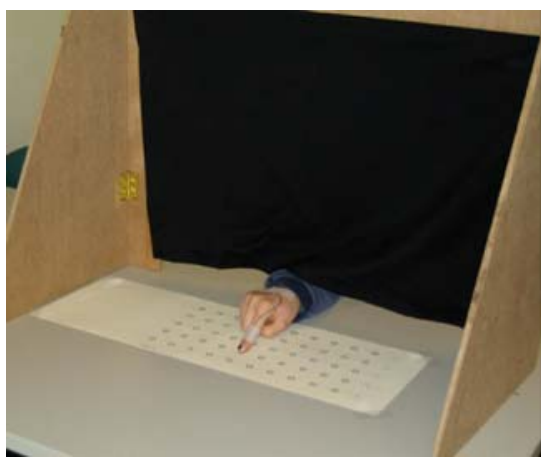

Fig. 1 Setup of experiment 1. Participants were sitting behind a table and a screen was placed between the participant and the stimuli (left panel). The right panel shows a participants' digit scanning a row of items on a sheet of swell paper. The swell paper contained five rows of

start moving their fingertip over the items. They could use any strategy that they wanted, including going back. As soon as they found the cross they had to lift their finger and put it back on the cross. If they could not find the target they had to lift their finger and keep it lifted.

\section{Analysis}

We analysed the velocity profiles of the individual movements in order to determine the total search time and analysed the individual movement trajectories to determine the time in contact with the items for each trial in each separation condition. The beginning of the search time was defined as the moment that the finger's velocity reached a threshold of 5\% of the maximal velocity between the starting point and the first item. The end of the search time was defined as the moment that the finger's vertical velocity was more than $1 \mathrm{~cm} / \mathrm{s}$, indicating that the participant had found the target. In order to quantify the time spent on each item we split each trial into movement time and time in contact with one of the items. A finger was regarded as being in contact with an item as long as there was some overlap between the finger and an item. Movement time was defined as the part of the search time during which the finger did not contact any of the items in the display. This way of defining contact gives us contact times that can be compared across the different conditions and separations, without taking the individual movement strategies that participants used into account. For each participant we determined the median search time and median time in contact with the items for each target position in each separation condition. The no-target trials were not analysed. We conducted an ANOVA (nine target positions, three separations) on the data set. We characterized the efficiency of the search by the slope of the search function: a linear regression of the search times as a function of target position.

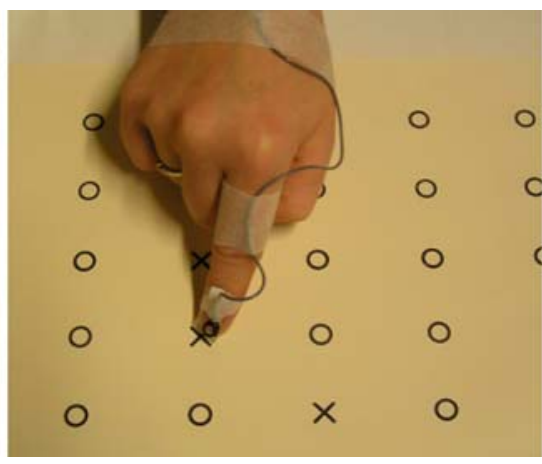

items, each row was used on a separate trial. The infrared emitting diode $(I R E D)$ on the nail of the index finger was used for recording its position

To compare the slopes of the search functions of this study with the slopes of previous static search experiments, ${ }^{1}$ we use the slope for the target absent condition from the previous studies. In the target absent condition of the static experiments participants have to check all items before they can decide whether the target is present or not. The display size in target absent trials in the earlier study is therefore comparable to the position of the target in the present study.

\section{Results}

The results are shown in Fig. 2a. Not surprisingly, when the position of the target was further away from the starting point, the search times were longer $(F(8,56)=229.16$, $P<0.001)$. In addition, the larger the separation between the items, the longer it took for the participant to find the target $(F(2,14)=50.41, P<0.001)$. We also found an interaction between position and separation $(F(16,112)=8.73$, $P<0.001)$. This interaction is captured by the different slopes of the search functions for the three separations: 0.52 s/item ('separation two'), 0.64 s/item ('separation four') and $0.80 \mathrm{~s} /$ item ('separation six').

When only considering the time in contact (Fig. 2b) there was no difference between the different separations $(F(2,14)=3.53, P=0.057)$, although we found an interaction effect $(F(16,112)=2.00, P<0.05)$. The slopes of the fitted functions for time in contact were: 0.52 s/item ('separation

\footnotetext{
${ }^{1}$ In earlier studies (Overvliet et al. 2007a, b) we used a static haptic search paradigm. Participants were sitting behind a curtain with their fingers at fixed positions. They had to lift their fingers and stimuli were put underneath their fingertips. After hearing a tone they had to simultaneously put their fingers down on the stimuli and then to lift the finger under which they thought there was a target present. If they did not feel a target, they had to lift all the fingers. They either used two, four or six fingers. The search time was measured from the moment the fingers touched the stimuli to the moment that the first finger was lifted.
} 


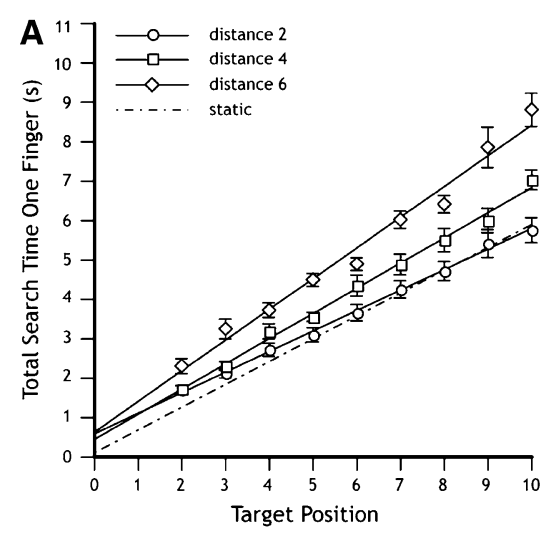

Fig. 2 Total search time (a) and time in contact with items (b) for the three different separation conditions in experiment 1 . Each point represents the mean of the median search times of eight participants. The error bars represent the standard error of this mean. The numbers on the

two'), $0.49 \mathrm{~s} /$ item ('separation four') and $0.51 \mathrm{~s} /$ item ('separation six'). The three functions overlap, as can be seen in Fig. 2b. The difference between the slope of the static search function $(0.58 \mathrm{~s} /$ item $)$ and the slope of the search function of the current experiment is not significant $(t=1.31)$. The above-mentioned interaction does not appear to result from a systematic difference in slope between the separations. Thus the systematic differences in search times between the separations must have been due to the movement time being longer for larger separations.

Examples of velocity profiles for the different separations are shown in Fig. 3. In 'separation two', where the finger was in contact with the next item as soon as it left an item, participants tended to move at a constant speed across the items. In 'separation six' they stopped to scan each item and moved very quickly between the items. In 'separation four' an intermediate strategy was used.

We checked whether the participants passed the target and then moved back. Although the participants made small movements from left to right over an item, they never moved back after passing an item, except for one participant who moved all the way back to the starting point if no target was present. Participants never moved to a next item when they were on the target.

\section{Discussion}

Separation has an influence on the slopes of the search functions: the greater the separation between the items the higher the slope of the search function. However, after subtracting the movement time no systematic relation between item separation and slope remained, indicating that the participant spends the same amount of time on each item, irrespective of the separation between the items. So participants apparently did not process information about an item during the movement to the next item.

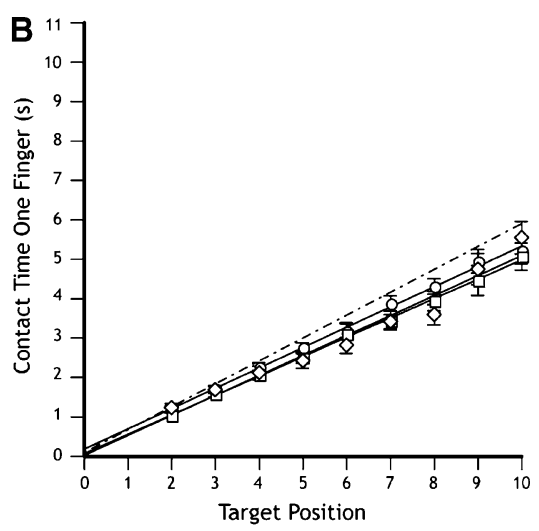

$\mathrm{x}$-axis are the number of items scanned. The line for the static task (dashed line) is the fit to the target absent condition from the Overvliet et al. (2007a) study, extrapolated to ten items
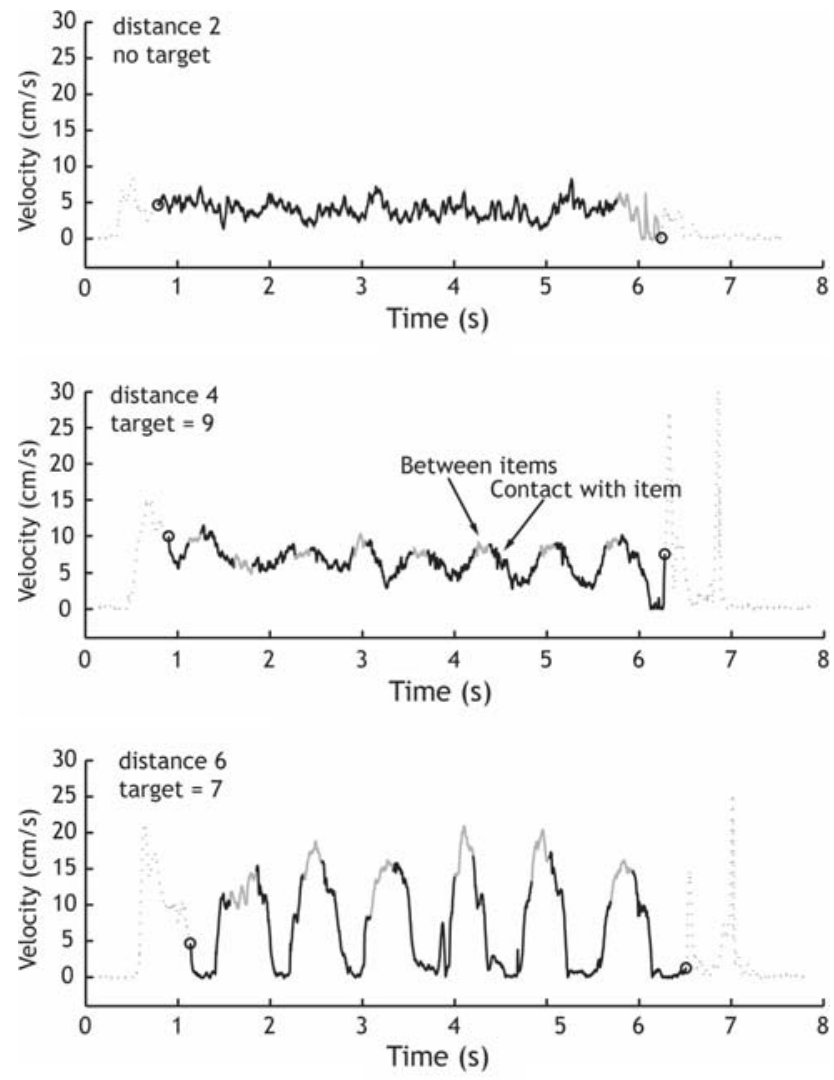

Fig. 3 Velocity profiles of typical individual trials of different participants of the three separation conditions in experiment 1 . The circles show the start and the end of the search. The light coloured parts of the curve are the periods in which the digit was not in contact with an item

The velocity profiles of individual trials in Fig. 3 suggest that the participant prefers to be on an item than in between items, but that there is no need to stand still on the item. In the 'separation two' condition the participant's finger is always in contact with an item, so he can use a constant speed to scan the items without losing any time, and this is 
what he does. In the 'separation six' condition the participant checks an item and then moves very quickly to the next item. The participant adjusts his movement strategy to the separation, so that he stays as long as necessary on an item and wastes as little time as possible between items.

We conclude that participants did not use the time between the items to process information about the item that they just passed. This indicates that the item must be processed completely before moving on to the next one. This is supported by the fact that participants did not pass the target and then go back to indicate that they had detected it, in contrast with the results found in visual search (Hooge and Erkelens 1996; Liesker et al. 2007). Although saccades are much faster and cost less energy than arm movements, our study is similar to visual search in that no new information can be gathered once the hand moves, but information could continue to be processed. The absence of return movements indicates that the tactile information was fully processed before the movement to the next item was initiated.

If we compare the current contact times with those of the search function obtained in a static haptic search experiment with exactly the same stimuli, we see that contact times in the moving search experiment were a little shorter than in the static search experiment (Fig. 2b). If we consider that movement planning, or cancelling a movement, takes at least $100 \mathrm{~ms}$, it becomes clear that the information must have been processed considerably faster in the current experiment than in the static search experiment. This suggests that feeling by moving one finger across an item may be more efficient than feeling by placing a fingertip on the item.

\section{Experiment 2}

We found that moving a finger across the items gives rise to faster information processing than touching the items simultaneously with multiple fingers. The question is whether this effect is caused by the movement of the finger or by the use of just one finger instead of multiple fingers. In earlier studies with similar stimuli it was shown that search times increased with the number of fingers used (Lederman et al. 1988; Lederman and Klatzky 1997; Overvliet et al. 2007a). However, when participants had to find a target between empty distractor elements, search times did not depend on the number of fingers that were used (Overvliet et al. 2007a).

We therefore wondered whether moving the fingers together, so that they could form one large sensor, would make the moving search task more efficient, because it would decrease the amount of time during which there was no contact with any item. There is evidence that exploring
2D objects with more than one finger decreases reaction times and increases the proportion of objects that are recognized (Jansson and Monaci 2004; Klatzky et al. 1993). However, a study on vibrotactile perception found the opposite result: a stimulus was recognised more often when presented to one finger than when presented to two fingers at the same time (Craig 1985).

Let us consider a few possible outcomes of our experiment. If the fingers form one sensor so that information is processed across them in parallel, search times will be shorter for large separations than in the one finger condition, because the time not in contact will decrease. If information cannot be processed in parallel over the fingers, there are two possibilities. One finger may be selected and information only processed at that finger, in which case the search times will be similar to those in the one finger condition. Alternatively, participants might analyse the information of all fingers serially, and thus switch regularly between fingers. This switching presumably takes time, so the search times will be longer than in the one finger condition.

\section{Method}

The same participants took part in this experiment as in experiment 1 . The equipment and method was also the same as in experiment 1 , except that now three fingers were used to scan the display. Participants had to align their index, middle and ring fingers and to scan the items with this row of fingers in a similar way as they did in experiment 1 (Fig. 4).

\section{Results}

The resulting haptic search functions are shown in Fig. 5 . The position of the target has an effect on the search time $(F(8,56)=64.93, P<0.001)$. The slopes of the functions

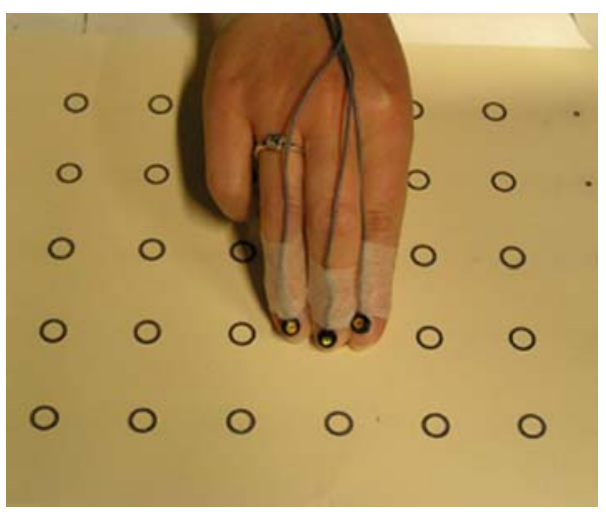

Fig. 4 The configuration of the three fingers used to scan the items in experiment 2 

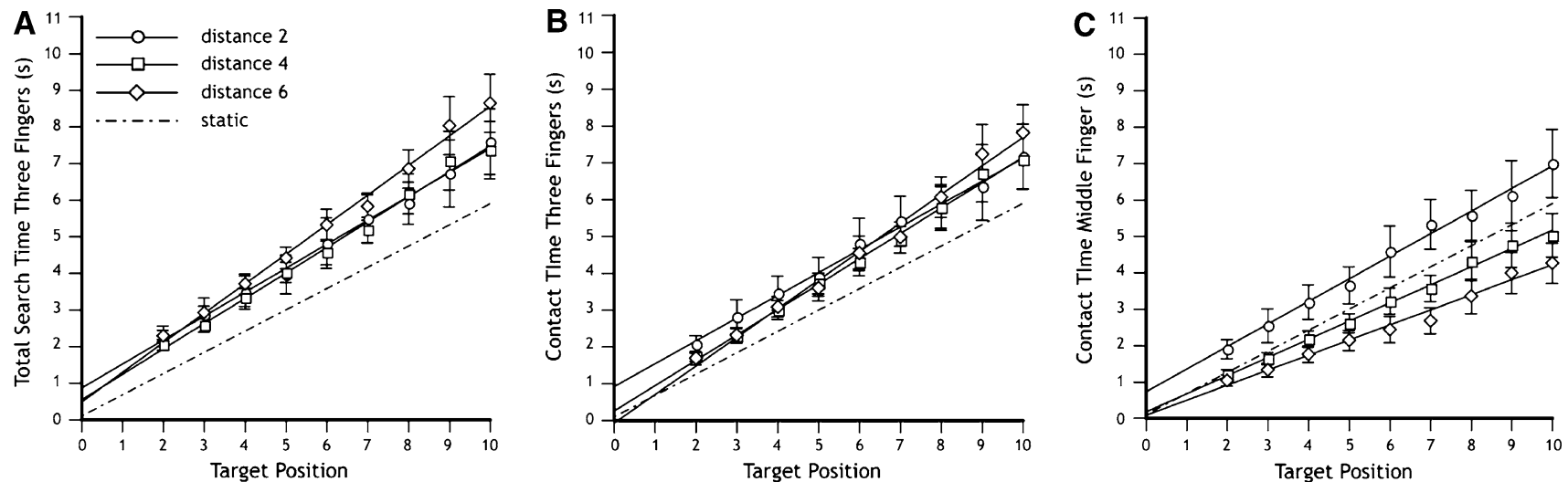

Fig. 5 Total search time (a), time in contact with the items (b) and contact time of the middle finger with the items (c) for the three different separation conditions in experiment 2. For further details see caption of Fig. 2

for the total search times (Fig. 5a) are $0.65 \mathrm{~s} /$ item for the 'separation two' condition, 0.69 s/item for the 'separation four' condition and $0.81 \mathrm{~s} /$ item for the 'separation six' condition. For 'separations four and six' the slopes of the total search time functions are the same as those for the same separations in experiment 1 . For 'separation two' the slope is higher in experiment 2 than in experiment 1 . If we look at the function for time in contact with any of the fingers (Fig. 5b) the slopes are higher in experiment 2 than in experiment $1(t=2.12, P<0.05)$. The time in contact per item is $0.62 \mathrm{~s}$ for 'separation two', $0.69 \mathrm{~s}$ for 'separation four', and $0.78 \mathrm{~s}$ for 'separation six'. Neither the average total search time, nor the contact time differed significantly between separations $(F(2,14)=2.60, \quad P=0.11, \quad$ and $F(2,14)=0.31, P=0.74$, respectively).

The middle finger was usually on the target when the participants lifted their fingers to indicate that the target had been found. We therefore also calculated the time that the middle finger was in contact with an item (Fig. 5c). This was on average $0.62,0.50$ and $0.41 \mathrm{~s} /$ item for 'separation two, four and six', respectively. In 'separation two' the slope is much higher than for the index finger in experiment 1 (Fig. 2b). In 'separation four' it is about the same and in 'separation six' the slope is lower. These lines do not have similar slopes, in contrast to what we found in experiment 1 , so participants were clearly not only using their middle finger.

The individual velocity profiles (Fig. 6) suggest that participants move much more slowly for 'separation two' than for the other separations. For 'separation four' there is a movement pattern that is similar to the pattern for 'separation two' in experiment 1. For 'separation six' there is a comparable velocity profile to that for 'separation four' in experiment 1 . In the velocity profile for 'separation two' that is shown in Fig. 6 one can see that the participant stops moving a few times. We therefore calculated the average amount of time that a participant stood
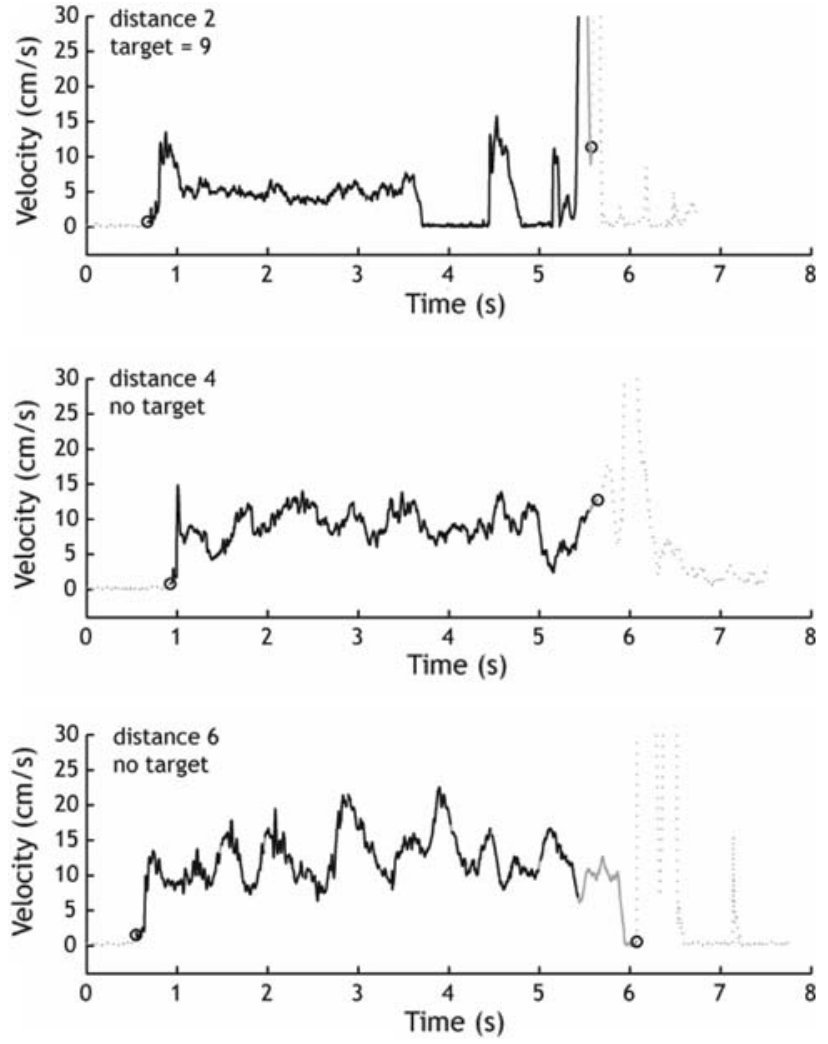

Fig. 6 Velocity profiles of typical individual trials of different participants of the three different separation conditions in experiment 2. For further details see caption of Fig. 3

still on an item for each condition in both experiments. This could be on any item, not necessarily the target. We defined standing still as a velocity of less than $1 \mathrm{~cm} / \mathrm{s}$. The resulting bar graph can be found in Fig. 7. In 'separation two' of experiment 2 , the amount of time that participants stopped moving was on average twice as high as in all the other conditions measured in experiments 1 and 2 (Fig. 7). 


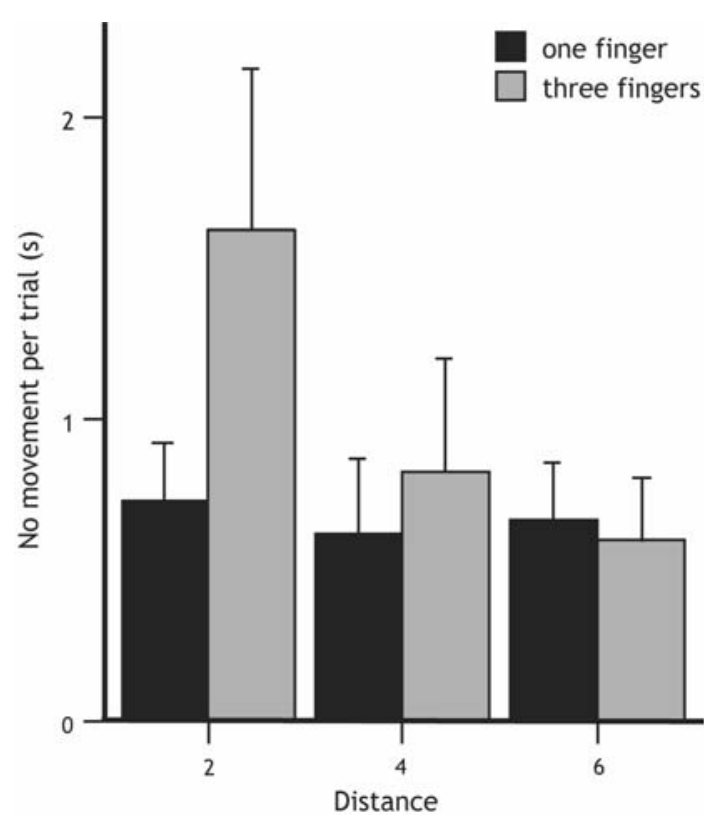

Fig. 7 Average amount of time that participants held their digits still (velocity $<1 \mathrm{~cm} / \mathrm{s}$ ) during a trial, when scanning with one finger (experiment 1) or with three fingers (experiment 2)

\section{Discussion}

The reason for processing times being shorter in a moving search experiment compared to a static experiment is evident from the current results. The time in contact in experiment 2 was higher than in experiment 1 for all separation conditions. So, the shorter processing times in experiment 1 compared to a static search experiment must have been the result of the use of one finger instead of multiple fingers. In experiment 2, processing of information, up to the moment that it is clear whether the item is a target or not, has to switch from one finger to another and this switching apparently costs time. This extends our earlier finding that critical shape information cannot be processed simultaneously across the fingers (Overvliet et al. 2007a, b). Another indication that searching with three fingers is much more difficult comes from the amount of time that participants stop moving. In 'separation two' of experiment 2 the total time that a participant was not moving is at least twice as high as in all other conditions. In 'separation two' there were always two items under the participant's fingertips. To be able to process all the information and switch the information processing from one finger to the other, the fingers had to slow down and even came to a stop several times.

The total search times for 'separations four and six' are more or less the same as in experiment 1 . The contact times are higher when using three fingers to scan the items. If participants had been able to combine the information from the three fingers as coming from one sensor, the contact times would have presumably been exactly the same as in experiment 1 . Therefore, the total search times would have been shorter in experiment 2 , because the time not in contact would be smaller due to the shorter distance as a result of a wider sensor. When looking at the results one can clearly see that this is not the case. The information from the different fingers is thus not combined as coming from one large sensor.

Our conclusion that searching with one finger is more efficient than searching with more fingers is largely in line with the results of Symmons and Richardson (2000). In their experiment participants could freely explore tactile line drawings. Most of the time their participants used only one finger to explore, which is the most efficient according to our conclusion. However, they report that for at least part of the exploration time, participants used multiple fingers close together that seemed to work as a single extended digit. A difference between their study and ours is that they used items that were bigger than the participants' fingers, while in our study participants had to explore items a little smaller than their fingertips. So, their participants might have used multiple fingers to be able to feel a complete item at once, which was always possible with a single finger in our experiment. We have some evidence that feeling a single item with several fingers can speed up static haptic search as well (Overvliet et al. 2007b).

\section{General discussion}

In the introduction we discussed possibilities to get more efficient tactile perception in a haptic search task. We argued that adding a separation between items could tell us something about the way that we process information about an item. We suggested that people do not necessarily need to stay in contact with an item to process information about it. In contrast with our expectations based on findings in visual search (Hooge and Erkelens 1996; Liesker et al. 2007), the results show that you finish tactile processing before you initiate a movement to the next item. This was revealed not only by the result that larger separations did not decrease the time that participants stayed in contact with the items, but also by the fact that participants never went on to the next item when they were in contact with the target. Moreover, participants adopt a strategy in which they spend as little time as possible between the items.

Performing the haptic serial search task with one moving finger was more efficient than performing a static search task. There is evidence that moving a finger or hand over a stimulus (or moving a stimulus over a finger or hand) results in shorter recognition times than presenting a static stimulus to a static hand or finger (Heller 1984; Phillips et al. 1983, see Introduction). Gibson (1962) also found that 
dynamic touch is better than static. He rotated objects on participants' fingertips and found that this resulted in a much better recognition rate than when the objects were pressed into the fingers. He argues that movement might help to filter out the noise, and therefore it might be easier to attend to the shape of the object. However, there are also studies in which no differences between static and dynamic touch were found. For example Pont et al. (1999) found that participants made the same systematic errors in curvature discrimination using dynamic and static touch, and concluded that similar mechanisms underlie both types of curvature discrimination. Levy et al. (2007) reported similar discrimination thresholds for the discrimination of twodimensional angles for dynamic and static touch. A difference between these studies and the studies in which a better performance is found for dynamic touch is the level of interpretation that is needed to perform the task. In the tasks in which better performance is found an object needs to be recognised. In the tasks in which equal performance was found, only a discrimination of simple shapes had to be made. Although our task needs very little interpretation, we did find a small advantage for moving the finger over the stimuli compared to static touch with multiple fingers. However, we already concluded in the discussion of experiment 2 that this advantage is most likely caused by the use of just one finger in the moving search task compared to the use of multiple fingers in the static task, rather than being caused by the motion.

In the second experiment we found that the processing time depended on how many fingers the participant used. When the participant used more than one finger at the same time, the task became much more difficult and the time in contact increased considerably. The time in contact in the previous static experiment (Overvliet et al. 2007a) is also larger than in experiment 1 . In both the static and the threefinger experiment the processing of information has to switch from one finger to another, while in experiment 1 , only one finger is used. This switching apparently costs time, so the time in contact with multiple fingers includes both switching time and processing time whereas in the one-finger experiment there is no need to switch, so this contact time only consists of processing time. Thus, the improvement in processing time in the first experiment compared to a static search task was caused by the use of one finger, the advantage of using only one finger being that you do not have to switch from one finger to the other, which apparently is not as easy (fast) as it seems.

\section{References}

Craig JC (1985) Attending to two fingers: two hands are better than one. Percept Psychophys 38(6):496-511

Gibson JJ (1962) Observations on active touch. Psychol Rev 69:477491

Heller MA (1984) Active and passive touch: the influence of exploration time on form recognition. J General Psychol 110(2d Half):243-249

Hooge IT, Erkelens CJ (1996) Control of fixation duration in a simple search task. Percept Psychophys 58(7):969-976

Jansson G, Monaci L (2004) Haptic identification of objects with different number of fingers. In: Ballesteros S, Heller MA (eds) Touch, blindness, and neuroscience. Uned Press, Madrid, pp 209-219

Klatzky RL, Lederman SJ (1995) Identifying objects from a haptic glance. Percept Psychophys 57(8):1111-1123

Klatzky RL, Loomis JM, Lederman SJ, Wake H, Fujita N (1993) Haptic identification of objects and their depictions. Percept Psychophys 54(2):170-178

Lederman SJ, Browse RA, Klatzky RL (1988) Haptic processing of spatially distributed information. Percept Psychophys 44(3):222232

Lederman SJ, Klatzky RL (1997) Relative availability of surface and object properties during early haptic processing. J Exp Psychol Hum Percept Perform 23(6): 1680-1707

Levy M, Bourgeon S, Chapman CE (2007) Haptic discrimination of two-dimensional angles: influence of exploratory strategy. Exp Brain Res 178(2):240-251

Liesker H, Smeets JBJ, Brenner E (2007) Simultaneous processing of visual information and planning of hand movements in a visuomanual search task. Acta Psychol (submitted)

Overvliet KE, Smeets JBJ, Brenner E (2007a) Parallel and serial search in haptics. Percept Psychophys (in press)

Overvliet KE, Mayer KM, Smeets JBJ, Brenner E (2007b) Haptic search is more efficient when the stimulus can be interpreted as consisting of fewer items. Acta Psychol. doi:org/10.1016/j.actpsy.2007.01.001

Phillips JR, Johnson KO, Browne HM (1983) A comparison of visual and two modes of tactual letter resolution. Percept Psychophys 34(3):243-249

Pont SC, Kappers AM, Koenderink JJ (1999) Similar mechanisms underlie curvature comparison by static and dynamic touch. Percept Psychophys 61(5):874-894

Purdy KA, Lederman SJ, Klatzky RL (2004) Haptic processing of the location of a known property: does knowing what you've touched tell you where it is? Can J Exp Psychol 58(1):32-45

Symmons M, Richardson B (2000) Raised line drawings are spontaneously explored with a single finger. Perception 29(5):621-626 